\title{
Clinical care or protocol violation?
}

As he slowly raised his hand, veterinarian John Collins knew that he would be the only IACUC member voting to withhold approval of the study proposed by Sandra Smith. The IACUC had already debated for nearly 20 minutes about the need for postoperative analgesia in the dogs that Smith was proposing to use in a survival surgical procedure. She had argued that there were ample scientific publications to show that analgesics could potentially interfere with the immune responses she was studying and that the surgical procedure itself (an ovariohysterectomy on 6-month-old animals) did not routinely require the use of postoperative analgesia when performed by private practice veterinarians. For his part, Collins argued that anesthesia, surgery and pain itself had immunosuppressive effects and that the failure of some private practitioners to align with modern veterinary practice was no reason to perpetuate unacceptable postoperative care. Smith persevered, reminding the IACUC that the surgery would be performed by the school's own veterinarians and that a high level of skill could therefore be anticipated. She said that, based upon what she was told by private practice veterinarians, the amount of anticipated pain would be minimal because it was not unusual for dogs to be alert, standing and even eating within a few hours of the procedure. Smith left the room, a vote was taken, and her protocol was approved as a USDA Category E study, that is, without the use of postoperative analgesia.

Soon afterwards, the study began and three of the first four dogs underwent the surgery with minimal clinically apparent aftereffects, as Smith had predicted. However, one animal was in obvious pain: the dog was lying on her side in the corner of her cage and whining long after the other animals were up and walking. Collins wanted to administer an analgesic, but he was cautioned by a colleague that using an analgesic would be a protocol violation. "That's ridiculous," said Collins. "At this point the animal is obviously in distress and anything I do to help her is considered clinical care. In fact, if I don't do something soon I might even be cited for not providing adequate veterinary care."

"Well, maybe" said his colleague, "but the IACUC approved this study to be performed without analgesia, and if you want to change that you have to go through the IACUC, whether you like it or not."

"I don't like it," countered Collins, "and I also don't like to see an animal in pain. If I go through the IACUC, what will that committee be able to do for the dog in any reasonable amount of time? I'm going to get some morphine. Let the chips fall wherever they fall."

Do you think Collins was right in assuming that the issue was one of providing appropriate clinical care to an animal that was clearly experiencing more pain than other animals undergoing the same procedure? Or do you think that Collins should have reported the problem to the IACUC and let the committee and the investigator decide on the appropriate course of action?

\section{RESPONSE}

\section{The bow and the lyre}

\section{Monte Matthews, BA, CPIA}

These chips, and all those of a similar nature, should always fall on Collins' side.

Both USDA regulations ${ }^{1}$ and $\mathrm{PHS}$ Policy ${ }^{2}$ require the appropriate use of sedatives, analgesics and anesthetics for procedures that may cause more than momentary pain or distress, unless withholding of such agents is scientifically justified in writing by the investigator. Although the principal investigator (PI), Smith, argues during the IACUC discussion of her protocol that there are "ample scientific publications to show that analgesics could potentially interfere with the immune responses she was studying," it isn't clear that this evidence was presented to the IACUC in writing as part of her protocol. We will assume that it was.

In addition to the scientific necessity, the withholding of analgesics was approved by the IACUC with the understanding that the surgical procedure would cause minimal postoperative pain, that it would not be unusual for the animals to "be alert, standing and even eating within a few hours of the procedure." However, there is no mention in this scenario of what to do if any of the animals exhibit more than this minimum amount of pain. Consequently, when one of the animals exhibited signs of more severe pain, beyond that which was expected and approved by the IACUC, then Collins' decision to relieve the animal's pain by administering an analgesic was his legal responsibility ${ }^{1,2}$.
USDA's definition of an attending veterinarian $(\mathrm{AV})$ is one who "has direct or delegated authority for activities involving animals" 1 and section 2.33(a)(2) of the Animal Welfare Act states, "Each research facility shall assure that the attending veterinarian has appropriate authority to ensure the provision of adequate veterinary care and to oversee the adequacy of other aspects of animal care and use." PHS Policy gives similar authority and responsibility to the $\mathrm{AV}$ and states that "medical care for animals will be available and provided as necessary by a qualified veterinarian" (IV.C.1.e.). Further, the Guide for the Care and Use of Laboratory Animals ${ }^{3}$ states, "A veterinary care program is the responsibility of the attending veterinarian," that "adequate veterinary care must be provided" and that the AV "must provide 